\title{
New Trends in Ultrasound-Guided Musculoskeletal Injuries Approaches
}

\author{
Bernáldez Domínguez Pedro ${ }^{1}$, Dallo Lazzarini Ignacio ${ }^{1}$
}

\section{Abstract}

The ultrasound not only allows us to diagnose musculoskeletal injuries but is also a fantastic tool to assist us when performing different therapies on the tissues. Multiple studies compare the classic blind versus ultrasound-guided infiltrations, with a significant difference in the results. In this article, we present the different indications for ultrasound-guided therapies, including ultrasound-guided local infiltration, percutaneous needle tenotomy, intracapsular hydrodilatation, hydrodissection, high-volume injection, percutaneous needle scraping and ultrasound-guided surgery (EAS). We describe the techniques, their advantages, and disadvantages, as well as possible complications. These procedures require a good learning curve, but once achieved, it will allow us to use them to obtain good clinical results, and in many cases avoiding the operating room. We have included these therapies in the so-called "SUBA Protocol" that includes the application of the concepts of modern Sports trauma, the use of musculoskeletal Ultrasounds, consider Biological therapies as one more tool in the therapeutic arsenal, and finally, the use of Arthroscopic surgeries, minimally invasive procedures with minor tissue damage.

Keywords: Musculoskeletal ultrasound, Ultrasound-guided therapies, Percutaneous needle tenotomy, Intracapsular hydrodilatation, Hydrodissection, Percutaneous needle scraping, Ultrasound-guided surgery, SUBA protocol

\section{Introduction}

Ultrasound is the complementary imaging test that has experienced the most significant progress in recent years in the specialty of orthopedic surgery and sports medicine.

It is a non-invasive diagnostic technique, where a probe (linear or warped) emits and receives ultrasounds, which penetrate to a certain depth the part of the body that we want to explore and allow us to see the normal anatomy as well as different pathologies of the musculoskeletal system [1]. The community of orthopedic surgeons is introducing themselves in this practice, and the use of ultrasound is increasing in their offices, emergency areas, and even in the operating room. And not only for its diagnostic utility but also for the possibility of performing ultrasound-guided therapeutic procedures, with direct and dynamic vision, on what we want to treat. In a recent article, we published what an ultrasound machine is like, its components, handling, and primary language, as well as the advantages of musculoskeletal ultrasound. We finalized it with its therapeutic utility and current indications in clinical practice [1]. There are some new technological advances, such as the three-dimensional ultrasound, but the current resolution is still suboptimal for clinical practice; elastography, which is applied to traumatized tissues, could help in the future the recovery of athletes [2], thus such as high-resolution doppler and doppler quantification, are allowing to complete the examination of the musculoskeletal lesion so that small lesions are now easily diagnosed [3].

Joint injections, punctures, and aspirations are used to reduce joint pain and decrease inflammation (Fig. 1). The effectiveness of these will decrease when they are placed inadvertently or in the wrong compartment [4].

This work aims to present the main indications for ultrasound-guided therapies (UGT), how to perform them, advantages and disadvantages, specific technical details, and possible complications. We will talk about the concept of ultrasound-guided surgery (UGS) and finally a review of the scant literature.

The UGT is a therapeutic gesture assisted by a high-frequency ultrasound by which we are going to administer a substance such as corticosteroids, local anesthetic, platelet-rich plasma, hyaluronic acid, or serum in a specific anatomical area, accurately and without extravasation, as well as performing minor additional therapeutic procedures such as perforations, tenotomies, exostectomy, fenestration, and aspiration of bruises or effusions.

It is a pioneering therapy with a bit of predicament, even in the specialty of orthopedic surgery. However, given the excellent clinical results, we are convinced that it will develop over the years. It is a fast, safe, low-cost therapy with a meager rate of complications, especially if we avoid surgery.
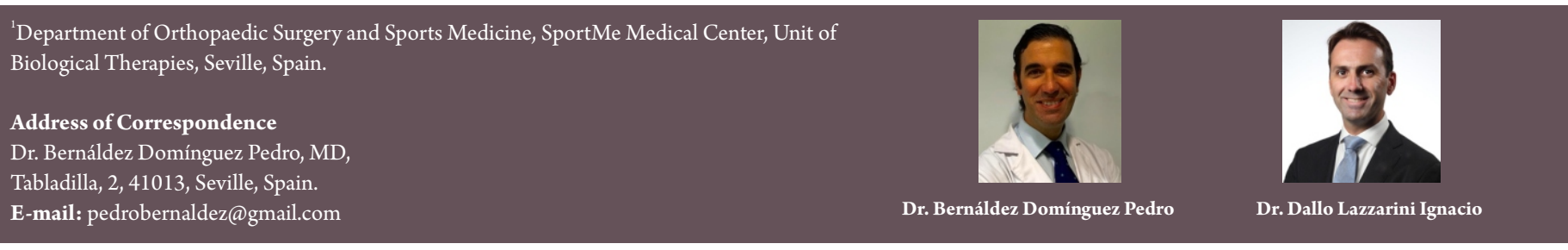

Submitted Date: 12 October 2021, Review Date: 14 Nevember 2021, Accepted Date: 17 November 2021 \& Published: 31 December 2021

(C) 2021 by Journal of Regenerative Science | Available on www.jrsonweb.com | DOI:10.13107/jrs.2021.v01.i01.017

This is an open access journal, and articles are distributed under the terms of the Creative Commons Attribution-NonCommercial-ShareAlike 4.0 License (https://creativecommons.org/licenses/by-nc-sa/4.0/), which allows others to remix, tweak, and build upon the work non-commercially, as long as appropriate credit is given and the new creations are licensed under the identical terms. 


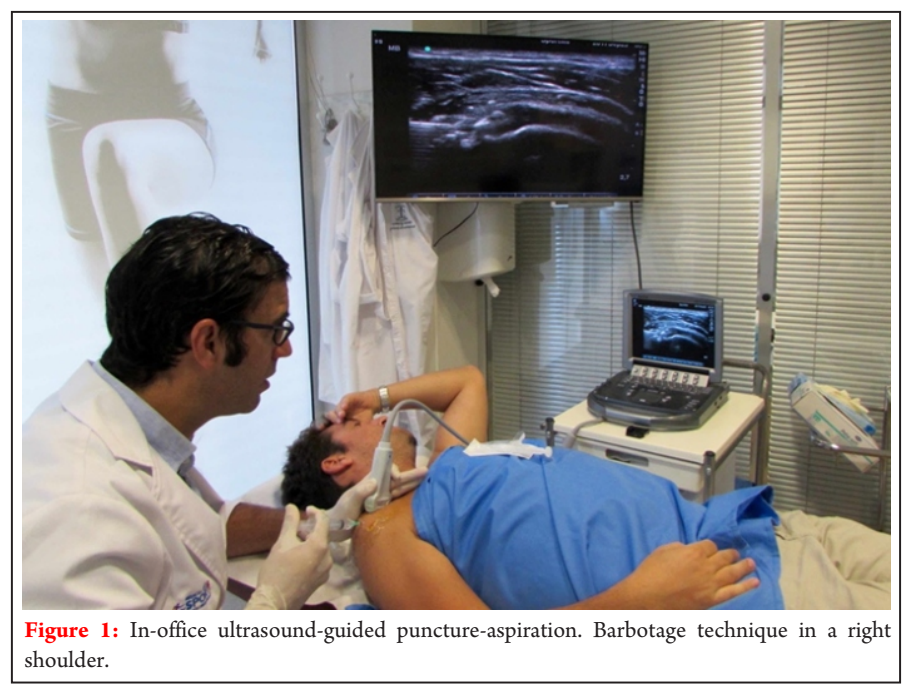

\section{Instruments}

It is minimal, just an ultrasound machine with a high-frequency linear probe or warped (for deeper structures), gloves, gel, needles of different sizes and lengths, and the medication to be used. In the case of UGS, different types of scalpels are needed (Fig. 2). Proper positioning of the doctor, respecting ergonomics, working comfortably in a stable environment are mandatory to avoid injuries. Sometimes, even the possibility of sharing the technique with the patient or family members.

In the upper extremity, ultrasound-guided injections have been shown to provide superior benefit over landmark-guided (blinded) injections in the glenohumeral joint, subacromial space, biceps tendon sheath, and hand and wrist joints.

Ultrasound-guided injections of the acromioclavicular and elbow joints are not more effective. However, ultrasound-guided injections in the knee, ankle, and foot are more effective than blinded injections $[4,5]$. In addition to developing the technique with greater accuracy, the ultrasound guide allows us to reduce the volume of medication to be used, providing more safety and avoiding damaging adjacent structures that could have adverse side effects such as intravascular injection or nerve injuries.

There is no conclusive evidence on the improved efficacy of ultrasound-guided hip injections, although blind injection is performed less frequently in this joint [4]. While current studies indicate that ultrasound guidance improves the efficacy and cost-effectiveness of many injections, these studies are limited and more research is needed.

Ultrasound-guided Therapie's Indications

Initially, we will diagnose the injury; we have to know what we are treating to cure it. Then, we will carry out minor procedures and administration of treatments precisely in the affected area, which optimizes the recovery of the injury. We avoid extravasation in one healthy anatomic area; for example, corticosteroids within a tendon can aggravate an injury.

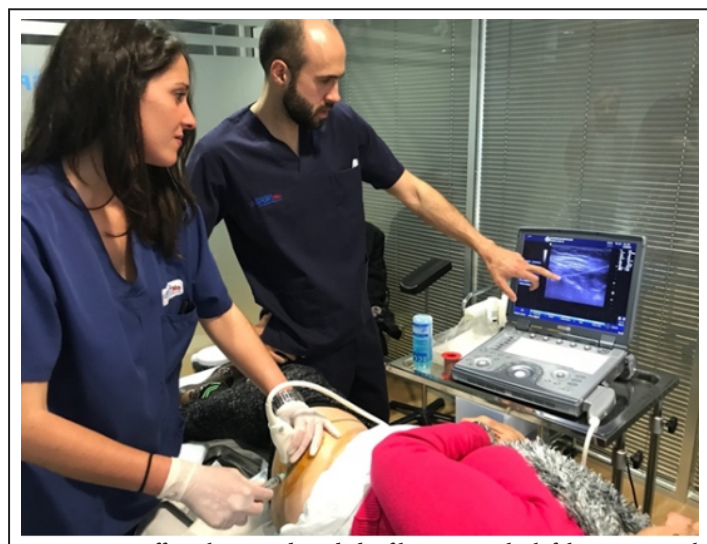

Figure 3: In-office ultrasound-guided infiltration on the left hip is essential to control the bevel of the needle during the procedure.

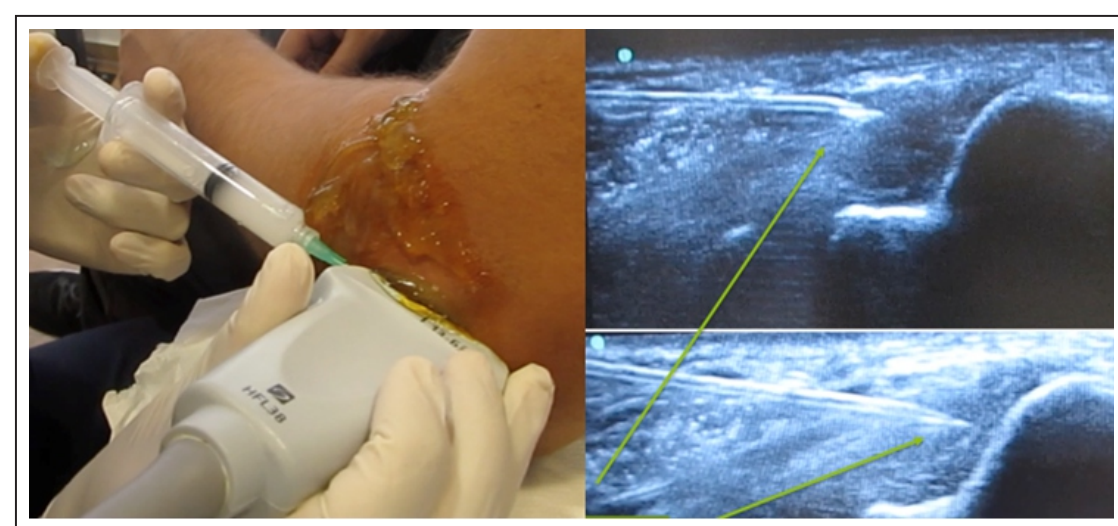

Figure 4: In-office ultrasound-guided percutaneous Extensor Carpis Radialis Brevis tenotomy in a left elbow.
Ultrasound is currently the only dynamic imaging test that allows us to interact with the patient and his injury directly. This fact is not provided by radiography, magnetic resonance, or tomography.

The most common injuries that we treat are inflammatory pathologies, such as tendinosis, enthesitis, bursitis, fascitis, mechanical pathologies like osteoarthritis, springs, muscular, meniscal or myofascial tears, neurological pathologies, canalicular, pseudosciatic syndromes, neuromas as well as capsulitis, stiffness, effusions, and calcifications (Table 1).

\section{Advantages and Disadvantages of UGT}

\section{Advantages}

The advantages of UGT are several since it is a harmless, minimally invasive, and generally painless technique, easily reproducible, and relatively inexpensive (Table 2 ).

We highlight the importance of being able to perform it in the same place of patient care and in real-time, in line with the trend of "Point of Care Ultrasound" (POCUS), without requiring referral to a radiology 


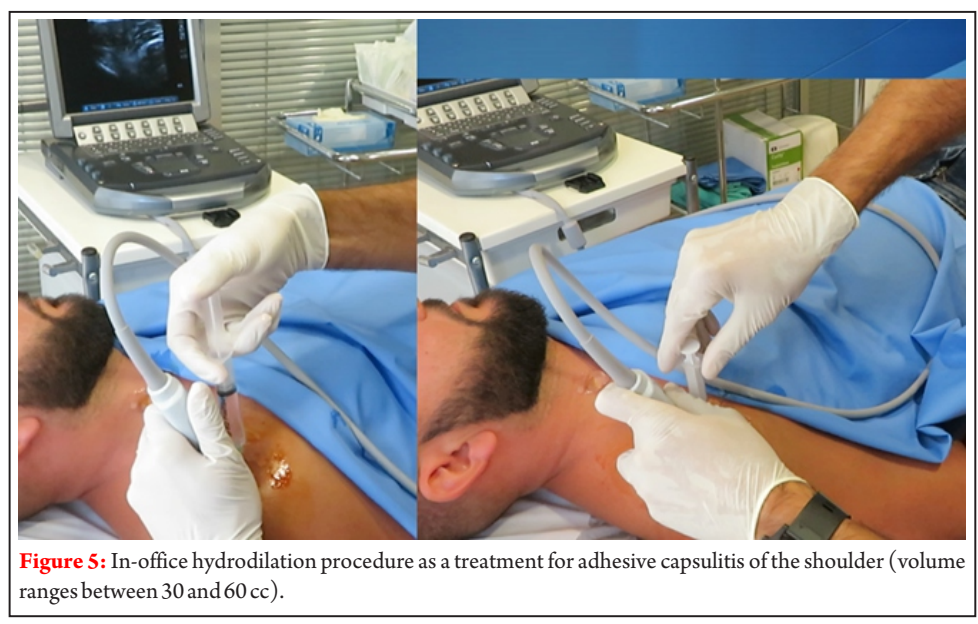

department, operating room or postpone the therapy.

\section{Disadvantages}

As it is an operator-dependent technique, it will require a specific skill when triangulating and performing the therapeutic act, and it has a learning curve that presents specific difficulties. However, it is known that orthopedic surgeons learn this technique relatively quickly due to their surgical practice and knowledge of the osteoarticular system's anatomy.

Complications include the possibility of infection in the case of not respecting the minimum aseptic measures. In our experience, the percentage after more than 1000 UGT is below $1 \%$. They are not superior to the blinded techniques.

\section{Procedures}

Under the concept of UGT, we include different procedures assisted by ultrasound where we highlight six techniques

1. Ultrasound-guided local infiltration

2. Percutaneous needle tenotomy (PNT) or fenestration

3. Percutaneous needle scraping (PNS)

4. Intracapsular hydrodilatation (ICH)

5. Hydrodissection (HD) or high-volume injection (HVI)

6.UGS.

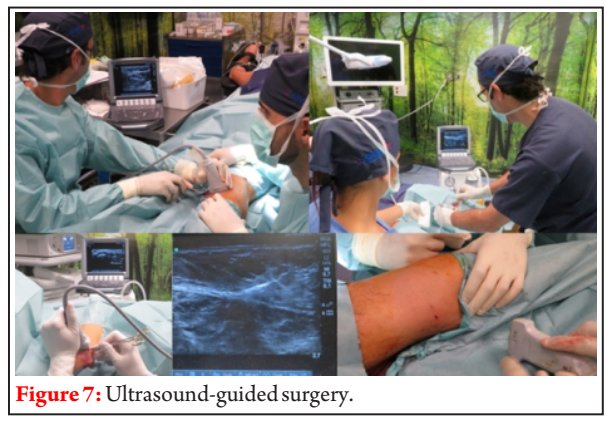

Ultrasound-guided infiltration (UGI)

We administer a drug in an anatomical area assisted by the ultrasound (Fig. 3). The most common are corticosteroids plus local anesthetic. However, we also inject plateletrich plasma (PRP), bone marrow aspirate concentrate, autologous micro fragmented adipose tissue, hyaluronic acid, botulinum toxin, and dextrose.

\section{Tenotomy with a percutaneous needle}

Tendinopathy is primarily a degenerative condition in the tendon, angiofibroblastic hyperplasia with a neurogenic inflammatory component that ultrasound can diagnose well.

Peck et al. [6] describe PNT as a treatment for tendinosis. With repetitive movements, we will make micro-perforations that will interrupt the chronic degenerative process, including the fibrotic tissue (scar) through bleeding that causes the release of growth factors and collagen formation.

The most frequent indications are extensor carpis radialis brevis tenotomy in epicondylitis (Fig. 4), adductor, and plantar fascia tenotomy. The bevel of the needle acts as a mini-scalpel. It breaks tissues causing a structured mechanical pathology such as springs, trigger fingers, even the Dupuytren pathology with the cordotomy technique. The procedure can be performed in the office; they are reasonably well tolerated with local anesthesia, and in well-selected cases, give good results.

\section{Scraping with a percutaneous needle}

It was described by Hall et al. as a treatment for chronic patellar tendinopathy when eccentric loading has failed and concerning the neurogenic influences involved [7]. It is an intervention aimed at cutting the neovessels and neural nerves using a scraping technique with the bevel of the needle from the posterior surface of the patellar tendon. It is also used on the Achilles tendon.

\section{ICH}

The etymology comes from Hydro: Water and Dilation: Expand.

Therefore, it consists of introducing water (saline solution) into an anatomical space, generally a joint limited in its movements, to expand its size or volume, allowing it to regain its functionality. It is a non-invasive, painless, and reproducible technique performed as an outpatient procedure, guided by ultrasound [8].

Its main indication is adhesive capsulitis, usually in the shoulder or ankle, presenting pain and reduced mobility symptoms. The procedure starts with the usual aseptic measures. Local anesthesia in the area and intra-articular is applied. A suprascapular nerve block may also be done. Introduce $30-60 \mathrm{cc}$ of saline solution in a pulsatile way using a $10 \mathrm{cc}$ syringe depending on the patient's type of joint (Fig. 5). Finally, we infiltrate corticosteroids (triamcinolone or dexamethasone). The duration of the technique is $10-15 \mathrm{~min}$, and the use of anesthesia makes it painless.

The results are sometimes appreciated immediately, especially the decrease in pain; after $5 \mathrm{~min}$, we begin to move the joint smoothly, gaining joint balance. It is mandatory to start with early physiotherapy to ensure that the patient recovers in a shorter time, reducing possible relapses.

\section{HD or HVI}

It consists of introducing a volume of saline solution (hydro) close to a nerve, muscle, or 


\begin{tabular}{|c|c|}
\hline Table 1: Common indications of ultrasound-guided therapies & Table 2: Advantages of ultrasound-guided injections \\
\hline Liquid aspiration & - It does not require hospital admission, and treatment \\
\hline - Bursitis & is performed on an outpatient basis \\
\hline - Ganglions & - It does not emit ionizing radiation (healthcare \\
\hline - Cysts & - It does not emit ionizing radiation (nealtncare \\
\hline - Drainage of Bruises & personnel and patients). \\
\hline - Spills (Morel Lavalle) & - The studies are dynamic and can be repeated to \\
\hline Infiltration of medication & follow the evolution of the injury \\
\hline - Platelet-rich plasma/stem cells & - Easy comparison with the contralateral limb and its \\
\hline $\begin{array}{l}\text { - Corticosteroids + Local Anesthetic } \\
\text { - Hyaluronic Acid }\end{array}$ & $\begin{array}{l}\text { Easy comparison with the contralateral ilmo and its } \\
\text { structure }\end{array}$ \\
\hline - Transcutaneous fluid therapy & - Localized and precise treatment. \\
\hline - Botulinum toxin & - It is done in real-time (POCUS) \\
\hline - Prolotherapy (Dextrose, etc.) & \\
\hline $\begin{array}{l}\text { Tenotomy or polectomy } \\
\text { - Spring finger }\end{array}$ & $\begin{array}{l}\text { Low cost (relatively cheap), especially if this is } \\
\text { going to avoid surgery: Optimization of healthcare costs }\end{array}$ \\
\hline - Epicondylitis (fenestration) & \\
\hline - Morton's neuroma & Table 3: Common indications of ultrasound-guided surgeries \\
\hline - Fasciectomy (medial twin, plantar, iliotibial band, etc.). & - Tendinopathy: Patellar, Quadriceps, Achilles \\
\hline - Scrapping or curettage & - Assistance to tendon ruptures: distal biceps, Achilles \\
\hline Biopsies & - Tendon lengthening and fascia: medial calf, Achilles, \\
\hline - Diagnosis of diseases & iliotibial girdle, compartment syndromes. \\
\hline ECO-assisted surgery (CEA) & - Cordotomies: Dupuytren's disease \\
\hline - Ganglions & - Lumps, spring fingers \\
\hline - Bursitis & - Cysts, Ganglions \\
\hline - Tendinosis & - Canalicular Syndromes: Carpal Tunnel, Cubital Tunnel, \\
\hline - Fasciitis & Sinus Tarsus, Morton Neuroma \\
\hline - - Calcifications & - Strange bodies \\
\hline - Neurolysis & - Bruising, Osteoscopy \\
\hline - Combination with arthroscopic procedures & - Calcium bursitis and tendinosis \\
\hline
\end{tabular}

tendon, at a specific pressure with the syringe to distend or dissect a body structure that may have adhesions, fibrosis, and be thickened (degenerated) $[9,10]$.

It is still an unusual practice even though it is being practiced in more and more centers. Our indications are fundamentally for nerve entrapment pathologies such as the sciatic nerve, carpal tunnel syndrome in the wrist (Fig. 6), radial or ulnar tunnel in the elbow.

This procedure can be done as many times as necessary to achieve complete separation ("detachment") of the nerve from the surrounding tissues and provide the desired relief. It has no contraindications, only severe systematic diseases. We must be careful not to puncture the nerve as it can cause a discharge and stabbing pain (neuropathy) that can last for several days.

\section{Puncture-aspiration(barbotage)}

Calcific tendinosis, more common in the shoulder, is caused by calcium deposits in the shoulder's rotator cuff. It is very painful. Thanks to the inclusion of ultrasound in our day-to-day life, direct diagnoses in the office are easy. In the same medical act, we can perform ultrasound-guided therapy through the puncture-aspiration technique (barbotage), washing and aspirating the tendons' calcification, breaking them, and extracting as much calcium as possible. The number of surgical indications has decreased thanks to the good results with this technique recovery.

Sometimes it goes hand in hand with the term ultra-mini-invasive surgery, although this is not necessarily always ultrasound-guided.

There are several indications to perform UGS; these are increasing year after year (Table 3).

This technique is not without complications; neurological and vascular structures can be damaged during the procedure.

\section{Discussion}

Under the name of UGT, we include various procedures that include UGI, PNT or fenestration, ICH, HD, or HVI, PNS and UGS.

They are procedures that require a good learning curve but, once achieved, will allow us to develop different techniques according to the type of pathology, obtain good clinical results, and on many occasions, avoid having to go through the operating room. Daley et al. [4] compare results of "blind" versus "ultrasound-guided" therapies where the use of images improved the precision of injection in the glenohumeral joint (95\% vs. $79 \%$ ), in the subacromial space ( $100 \%$ vs. $63 \%)$, in the acromioclavicular joint (ACJ) (100\% vs. $45 \%$ ) and the knee (99\% vs. $79 \%$ ).

They also state a statistically higher accuracy rate with the posterior approach than in the glenohumeral joint ( $85 \%$ vs. $45 \%$ ). The injection site selection did not affect the accuracy of the subacromial space, ACJ, elbow, or knee.

In a prospective, randomized, nonobservational study conducted by SabetiAschraf et al. [14] on 60 cadavers, 120 ACJs, six physicians with three different levels of experience infiltrated $20 \mathrm{ACJs}$ each. Half of them infiltrated after palpation of the joint space and the other half with ultrasound. Controls were carried out before and after the infiltration by an independent radiologist. The difference between the two groups was significant $(\mathrm{P}=0.009)$. They conclude by stating that UGI in the ACJs is significantly more accurate than the blinded technique. This method is simple, efficient, and can be applied by therapists of all levels of experience.

Berkoff et al. [15] confirm the greater effectiveness in ultrasound-guided knee infiltrations compared with the anatomical reference $(96.7 \%$ vs. $81 \%)$ as well as in the shoulder ( $97.3 \%$ vs $65.4 \%$ ), both with $\mathrm{P}<$ 
0.001 . Very similar results are published in the studies of Aly et al. [16] and Wu et al. [17] regarding the shoulder joint.

The same conclusion was reached in the hip joint in the study by Hoeber et al. [18], where he compared 136 UGIs (in 4 studies) versus 295 blind (5 studies); in the first, the effectiveness was $100 \%$ versus $72 \%$ with $\mathrm{P}<$ 0.0001 .

Evidence also shows that less experienced physicians can improve their accuracy with the help of ultrasounds [6]. Continuous study of ultrasound-guided injections will help guide medical practice. Our team has performed these techniques with good results for the past 10 years and presented them in many scientific meetings.

As complications of the technique, highlight the possibility of an infection in the case of not respecting the minimum aseptic measures. In our experience, the percentage is below $1 \%$.

We have included these therapies in the socalled "SUBA Protocol" that includes the application of the concepts of modern Sports trauma, the use of musculoskeletal Ultrasounds, consider Biological therapies as one more tool in the therapeutic arsenal, and finally, the use of Arthroscopic surgeries, minimally invasive procedures with minor tissue damage [19].

\section{Conclusion}

Nowadays, clinicians can better evaluate soft tissue pathology such as tendinopathy, bursitis, fasciitis, effusions in the same consultation, in real-time, with highresolution MSKultrasound images.

According to current data, UGT appear to be safe and effective, and this will increase as technology improves and the availability of ultrasound increases. They are generally more accurate than blinded injections. However, its evidence is still limited by its size and quality. Further research with prospective, randomized controlled trials with larger sample sizes and perhaps compared to other treatments further defines their safety, efficacy, cost-effectiveness, and role in treating musculoskeletal pathology.

Declaration of patient consent: The authors certify that they have obtained all appropriate patient consent forms. In the form, the patient has given his consent for his images and other clinical information to be reported in the Journal. The patient understands that his name and initials will not be published, and due efforts will be made to conceal his identity, but anonymity cannot be guaranteed.

\section{Conflicts of Interest: Nil. Source of Support: None.}

\section{References}

1. Domínguez B, Martos TA. El ecógrafo: El fonendo del Traumatólogo: Utilidad diagnostica y terapéutica. Rev S Traum Ort 2017;34:17-26.

2. De Zordo T, Lill SR, Fink C, Feuchtner GM, Jaschke W, Bellmann-Weiler $R$, et al. Real-time sonoelastography of lateral epicondylitis: Comparison of findings between patients and healthy volunteers. AJR Am J Roentgenol 2009;193:180-5.

3. Nazarian LN. The top 10 reasons musculoskeletal sonography is an important complementary or alternative technique to MRI. AJR Am J Roentgenol 2008;190:1621-6.

4. Daley EL, Bajaj S, Bisson LJ, Cole BJ. Improving injection accuracy of the elbow, knee, and shoulder: Does injection site and imaging make a difference? A systematic review. Am J Sports Med 2011;39:656-62.

5. Daniels EW, Cole D, Jacobs B, Phillips SF. Existing evidence on ultrasound-guided injections in sports medicine. Orthop J Sports Med 2018;6:2325967118756576.

6. Peck E, Jelsing E, Onishi K. Advanced ultrasound-guided interventions for tendinopathy. Phys Med Rehabil Clin NAm 2016;27:733-48.

7. Hall MM, Rajasekaran S. Ultrasound-guided scraping for chronic patellar tendinopathy: A case presentation. PMR 2016;8:593-6.

8. Patel R, Urits I, Wolf J, Murthy A, Cornett EM, Jones MR, et al. A comprehensive update of adhesive capsulitis and minimally invasive treatment options. Psychopharmacol Bull 2020;50 4 Suppl 1:91-107.

9. Lam KH, Hung CY, Chiang YP, Onishi K, Su DC, Clark TB, et al. Ultrasound-guided nerve hydrodissection for pain management: rationale, methods, current literature, and theoretical mechanisms. J Pain Res 2020;13:1957-68.

10. Boesen AP, Hansen R, Boesen MI, Malliaras P, Langberg H. Effect of high-volume injection, platelet-rich plasma, and sham treatment in chronic midportion achilles tendinopathy: A randomized double-blinded prospective study. Am J Sports Med 2017;45:2034-43.
11. Tafti D, Byerly DW. Ultrasound Guided Barbotage. Treasure Island, FL: StatPearls Publishing; 2021.

12. Henning PT, Yang L, Awan T, Lueders D, Pourcho AM. Minimally invasive ultrasound-guided carpal tunnel release: Preliminary clinical results. J Ultrasound Med 2018;37:2699-706.

13. Gao L, Madry H, Chugaev DV, Denti M, Frolov A, Burtsev M, et al. Advances in modern osteotomies around the knee: Report on the association of sports traumatology, arthroscopy, orthopaedic surgery, rehabilitation (ASTAOR) Moscow International Osteotomy Congress 2017. J Exp Orthop 2019;6:9.

14. Sabeti-Aschraf M, Lemmerhofer B, Lang S, Schmidt M, Funovics PT, Ziai $P$, et al. Ultrasound guidance improves the accuracy of the acromioclavicular joint infiltration: A prospective randomized study. Knee Surg Sports Traumatol Arthrosc 2011;19:292-5.

15. BerkoffDJ, Miller LE, Block JE. Clinical utility of ultrasound guidance for intra-articular knee injections: A review. Clin Interv Aging 2012;7:89-95.

16. Aly AR, Rajasekaran S, Ashworth N. Ultrasound-guided shoulder girdle injections are more accurate and more effective than landmark-guided injections: A systematic review and meta-analysis. $\mathrm{Br} J$ Sports Med 2015;49:1042-9.

17. $W u$ T, Song $H X$, Dong $Y, L i ~ J H$. Ultrasound-guided versus blind subacromial-subdeltoid bursa injection in adults with shoulder pain: $A$ systematic review and meta-analysis. Semin Arthritis Rheum 2015;45:3748.

18. Hoeber S, Aly AR, Ashworth N, Rajasekaran S. Ultrasound-guided hip joint injections are more accurate than landmark-guided injections: A systematic review and meta-analysis. Br J Sports Med 2016;50:392-6.

19. Domínguez MP. Bridging the Gap between Surgical and Conservative Treatment. The SUBA Protocol (Sports, Ultrasound, Biologics, Arthroscopy). TOBI (The Orthobiologic Institute) Conference; 2021.

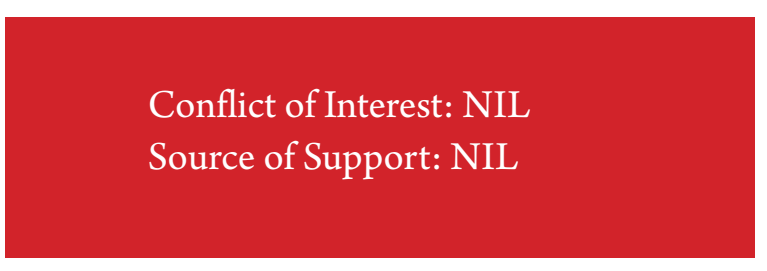

\section{How to Cite this Article}

Pedro BD, Ignacio DL | New Trends in Ultrasound-Guided Musculoskeletal Injuries Approaches | Journal of Regenerative Science | December 2021; 1(1):30-34. 